\title{
CONCEPÇÕES DE ENFERMEIROS SOBRE A POLÍTICA NACIONAL DE ATENÇÃO INTEGRAL À SAÚDE DO HOMEM
}

\author{
CONCEPTS OF NURSES ON THE NATIONAL MEN'S HEALTH CARE POLICY \\ CONCEPCIONES DE ENFERMEROS SOBRE LA POLÍTICA NACIONAL DE ATENCIÓN INTEGRAL \\ A LA SALUD DEL HOMBRE
}

\author{
Daiane Cristina Teixeira ${ }^{1}$ \\ Daiane Kutszepa Brambilla ${ }^{2}$ \\ Edlamar Kátia Adamy ${ }^{3}$ \\ Ivete Maroso Krauzer ${ }^{4}$
}

Resumo O estudo objetivou conhecer a implantação da Política Nacional de Atenção Integral à Saúde do Homem na ótica dos enfermeiros. Trata-se de uma pesquisa qualitativa exploratória e descritiva, em que foram entrevistados 17 enfermeiros dos municípios da Associação dos Municípios do Entre Rios, em Santa Catarina. Após análise dos dados, considerou-se que há fragilidades no conhecimento da Política Nacional de Atenção Integral à Saúde do Homem, bem como em ações realizadas nos municípios. A saúde do homem está centrada no modelo biomédico de atenção à saúde. Conclui-se que há necessidade de capacitação dos profissionais para o desenvolvimento da política de acordo com os seus pressupostos.

Palavras-chave saúde do homem; políticas públicas; atenção básica.
Abstract This article focuses on the implementation of the National Men's Health Care Policy from the nurses' perspective. It is an exploratory and descriptive qualitative study in which 17 nurses from the municipalities of the Entre Rios Municipalities Association, in Santa Catarina (Brazil), were interviewed. After analyzing the data, it was concluded that there are knowledge gaps both concerning the National Men's Health Care Policy and on the actions carried out at the municipalities. Men's health is focused on the biomedical health care model. It is concluded that there is need to train the professionals in order to attain the development of the policy pursuant to its assumptions. Keywords human health; public policy; primary care. 
Em 2006 foi criada a Política Nacional de Atenção Básica (PNAB), que revisou e adequou as normas para a Atenção Básica no Brasil, também conhecida como Atenção Primária em Saúde. Esta se caracteriza por ações em saúde de caráter individual e coletivo, que abrangem promoção da saúde, prevenção de doenças, diagnóstico, tratamento, reabilitação e manutenção da saúde (Brasil, 2006a).

$\mathrm{Na}$ PNAB foram determinadas as responsabilidades dos municípios e dos profissionais da atenção básica, cabendo ao enfermeiro prestar assistência integral às pessoas e à família na lógica da Estratégia Saúde da Família (ESF), além de realizar as consultas e os procedimentos de enfermagem; coordenar; gerenciar e planejar as ações dos agentes comunitários de saúde (ACS); organizar e ordenar grupos específicos e famílias em risco na região de sua atuação; efetivar e supervisionar atividades de educação permanente e fazer gerenciamento de insumos necessários à unidade (Brasil, 2006b).

A Política Nacional de Atenção Integral à Saúde do Homem (PNAISH) foi instituída pela portaria n. 1.944, de 27 de agosto de 2009, do Gabinete do Ministério, tendo como objetivo promover a melhoria das condições de saúde da população masculina no Brasil, contribuindo, de modo efetivo, para a redução da morbidade e mortalidade dessa população, por meio do enfrentamento racional dos fatores de risco e mediante a facilitação ao acesso às ações e aos serviços de assistência integral à saúde (Brasil, 2008).

A PNAISH foi formulada com foco principal para a população de homens adultos, na faixa de 20 a 59 anos. A política não desconsidera as outras faixas etárias, mas prioriza suas ações a esse recorte etário, pois se acredita que crianças, adolescentes e idosos são Assis tidos mais frequentemente nos serviços de saúde (Pirolo et al., 2009).

A PNAISH está alinhada e em consonância com os princípios da PNAB. Pretende fortalecer ações e serviços em redes, sobretudo qualificar a atenção básica, para que não se restrinja somente à recuperação da saúde, garantindo a promoção da saúde e a prevenção de agravos evitáveis (Conselho Nacional de Secretários de Saúde, 2010).

Oferecer e facilitar o acesso da população masculina a um conjunto de ações de promoção, prevenção, assistência e recuperação da saúde, executadas nos diferentes níveis de assistência, são objetivos da PNAISH. Entretanto, a prioridade na saúde do homem é a atenção básica, ou seja, promoção da saúde e prevenção dos agravos por meio da ESF.

A expectativa de vida da população masculina é menor do que a feminina, e na análise do perfil de morbimortalidade dos homens, nota-se que estes morrem mais pelas principais causas de morte do que as mulheres. Historicamente, quanto aos agravos, os homens sempre apresentaram idade média inferior às mulheres, o que é corroborado por Lyra e colaboradores 
(2010, p. 110), segundo os quais, ao se analisar "o diferencial de mortalidade segundo sexo e idade, percebe-se nitidamente uma sobremortalidade masculina em todos os grupos etários, principalmente nos mais jovens". Assim, parece ser importante que as equipes de ESF realizem ações para modificar essa realidade.

Incluir os homens nos serviços de saúde para que se sintam acolhidos e parte integrante do sistema de saúde é um trabalho a ser desenvolvido na atenção básica, pois é um cenário privilegiado para o desenvolvimento de práticas, devido à sua maior proximidade com a população e à ênfase nas ações preventivas e de promoção à saúde (Pinafo et al., 2011). Por meio de ações educativas, os homens podem ser sensibilizados para a responsabilidade de cuidar de si e dos outros, o que facilita o rompimento da barreira existente entre homens e unidades de saúde (Brasil, 2008).

A implantação dessa política deverá ocorrer de forma integrada às demais políticas existentes, obedecendo a uma lógica hierarquizada de atenção à saúde, priorizando a Atenção Básica como porta de entrada de um sistema de saúde universal, integral e equânime (Brasil, 2008). Nesse sentido, o Ministério da Saúde (MS) cumpre seu papel, possibilitando o reconhecimento e o respeito à ética e aos direitos do homem e obedecendo às suas peculiaridades socioculturais (Reichert e Andrioli, 2010).

A maioria das políticas governamentais implantadas ainda privilegia a saúde das mulheres e a estratificação por grupos prioritários, como crianças menores de um ano, gestantes e idosos, dentre outros. Isto se evidencia pelos estudos publicados na literatura que demonstram mais as experiências das mulheres (Medrado e Lyra, 2008).

Os municípios brasileiros buscam conhecer essa política para adequar suas ações a fim de atender a população masculina, pois a atenção à saúde do homem faz parte dos objetivos do Pacto pela Saúde. Este pacto é um documento criado pelo MS, juntamente com o Conselho Nacional de Secretários de Saúde e o Conselho Nacional de Secretarias Municipais de Saúde, que estabeleceu responsabilidades entre as diferentes instâncias de poder no campo de gestão e assistência à saúde. Uma das prioridades pactuadas é a atenção à saúde do homem, pela qual, assinando esse documento, os municípios deveriam atuar (Brasil, 2006a).

No estado de Santa Catarina, essa política tem seguido o planejamento do Ministério da Saúde, cuja área técnica de saúde do homem indicou, em 2010, os municípios de Joinville, Florianópolis, Lages e Chapecó para implantarem a PNAISH.

Os dados apresentados neste artigo são resultados de uma pesquisa desenvolvida como trabalho de conclusão do curso de enfermagem da Universidade do Estado de Santa Catarina, que procurou identificar as concepções 
dos enfermeiros acerca da PNAISH, bem como conhecer as ações desenvolvidas nos municípios. Entende-se que, para a implantação da PNAISH, os enfermeiros devem conhecer a proposta para poder estabelecer estratégias de atendimento a determinada população.

A atuação do enfermeiro como membro da equipe multiprofissional da atenção básica, junto à ESF, visa resolver ou minimizar os problemas mais incidentes e prevalentes na população, intervindo por meio da promoção da saúde e prevenção de doenças. Esse profissional deve atuar para melhorar a saúde do homem, tendo em vista o perfil de morbimortalidade dessa parcela da população e da sua menor expectativa de vida em relação às mulheres (Conselho Federal de Enfermagem, 2007).

$\mathrm{Na}$ área da enfermagem, um estudo sobre essa temática se justifica, pois o enfermeiro é um dos responsáveis pelas ações desenvolvidas e, consequentemente, pela consolidação dessa política.

\section{Percurso metodológico}

Trata-se de uma pesquisa qualitativa de caráter exploratório e descritivo. ${ }^{5}$ Os sujeitos foram os enfermeiros que trabalhavam na ESF de 17 municípios pertencentes à Associação dos Municípios do Entre Rios (Amerios), Santa Catarina. Fazem parte da Amerios os seguintes municípios: Bom Jesus do Oeste, Caibi, Cunha Porã, Cunhataí, Flor do Sertão, Iraceminha, Maravilha, Modelo, Palmitos, Riqueza, Romelândia, Saltinho, Santa Terezinha do Progresso, São Miguel da Boa Vista, Saudade, Tigrinhos e Campo Erê.

A coleta de dados foi realizada nos meses de julho e agosto de 2011 pelas pesquisadoras, em visitas previamente agendadas aos sujeitos da pesquisa. As entrevistas foram gravadas e norteadas por um instrumento com questões semiestruturadas. Após a coleta dos dados, as entrevistas foram transcritas e categorizadas para a análise dos resultados.

As categorias analisadas foram: conhecimento dos enfermeiros em relação à PNAISH e seus objetivos; papel do enfermeiro diante da PNAISH; fatores que interferem na resistência dos homens em procurar os serviços de saúde; perfil epidemiológico da população masculina segundo os enfermeiros e recursos financeiros direcionados à saúde do homem.

Essa pesquisa foi aprovada com o número 23/2011 pelo Comitê de Ética em pesquisa da Universidade do Estado de Santa Catarina, obedecendo à resolução n. 196/1996 do Conselho Nacional de Saúde. Para a identificação dos sujeitos, utilizou-se a letra ' $E$ ' para enfermeiro seguida pelos números de 1 a 17 (por exemplo: E5). 


\section{Resultados e discussão}

Os resultados obtidos foram explanados de acordo com as categorias citadas na metodologia. Desta forma, foi possível analisar e interpretar as ideias dos entrevistados, a fim de alcançar os objetivos propostos por este trabalho. $\mathrm{O}$ tempo de atuação dos enfermeiros entrevistados obteve uma média de seis anos e seis meses, sendo que o tempo maior de experiência profissional foi de 25 anos, e a menor, de quatro meses.

\section{Conhecimento dos enfermeiros em relação à Política Nacional de Atenção Integral à Saúde do Homem e seus objetivos}

Quando questionados sobre o conhecimento em relação à PNAISH, 11 enfermeiros relataram conhecer a política, quatro informaram conhecer parcialmente, e dois enfermeiros responderam não ter conhecimento sobre o tema. Com base nessas informações, foi possível identificar que os enfermeiros têm conhecimento sobre o tema saúde do homem. Nos depoimentos, percebe-se que esse conhecimento está relacionado à prática assistencial desenvolvida, pois eles discorreram sobre os atendimentos diários relacionados à saúde do homem ou fizeram um comparativo entre saúde do homem e saúde da mulher. Pode-se afirmar que o conhecimento voltado para os pressupostos da PNAISH foi evidenciado em três dos 11 enfermeiros que diziam conhecer a política.

Para um dos entrevistados, os profissionais de saúde desconhecem integralmente a PNAISH: “Essa política é um programa novo que poucas pessoas têm conhecimento dessa área, inclusive os profissionais de saúde" (E16).

$\mathrm{Na}$ análise das entrevistas, ficou evidente a preocupação dos enfermeiros com a incidência das neoplasias de próstata, citada por oito dos entrevistados $(47 \%)$. No entanto, a política preconiza ações prioritárias na faixa etária dos 20 aos 59 anos, e a faixa etária mais acometida por esse tipo de câncer está entre os homens com mais de 50 anos (Brasil, 2008). Assim, torna-se necessário despertar as equipes de saúde em relação às demais causas de morte na faixa etária preconizada pela PNAISH.

Dados estatísticos apontam ainda as causas externas como a primeira causa de morte na população masculina, na faixa etária preconizada pela política, seguida pelas doenças do aparelho circulatório, os tumores, as doenças do aparelho digestivo e as do aparelho respiratório, entre outras (Brasil, 2008). Nesse caso, identifica-se uma necessidade imediata da realização de ações relacionadas a causas externas, pois é a maior representatividade de morte precoce no homem, principalmente na faixa dos 20 aos 40 anos, faixa de maior produtividade do homem. 
No estudo de Julião e Weigelt (2011) sobre atenção à saúde do homem em unidades de ESF, foi possível identificar que há fragilidades nas informações epidemiológicas fornecidas pelos enfermeiros em relação à PNAISH - o que pode ser devido a falhas no repasse de informações por parte das secretarias de saúde ou falta de sensibilização dos profissionais em se aperfeiçoar e implementar ações em relação à saúde do homem.

Quanto aos objetivos da PNAISH, as respostas dos enfermeiros são diversas. A maioria discorreu sobre prevenção de doenças (13 enfermeiros, 76,4\%). Outras respostas apontaram a diminuição de índices de câncer de próstata (oito enfermeiros, 47\%); diminuição do perfil de morbimortalidade (dois enfermeiros, 11,8\%); relação da saúde do homem com o acesso ao serviço (quatro enfermeiros, 23,5\%); atendimento integral (um enfermeiro, 5,9\%); aumento da expectativa de vida dos homens (um enfermeiro, 5,9\%). Um enfermeiro relacionou o objetivo com a construção de dados epidemiológicos.

O objetivo geral da PNAISH, segundo o Ministério da Saúde, é:

Promover a melhoria das condições de saúde da população masculina do Brasil, contribuindo, de modo efetivo, para a redução da morbidade e mortalidade desta população, através do enfrentamento racional dos fatores de risco mediante a facilitação ao acesso, às ações e aos serviços de assistência integral à saúde do homem (Brasil, 2008, p. 31).

No que se refere ao conhecimento dos objetivos da PNAISH, o enfoque das falas dos enfermeiros é a prevenção das doenças (13 enfermeiros). Destes, oito focalizaram os objetivos na prevenção do câncer de próstata e não evidenciaram claramente os objetivos da política, que prevê a assistência integral à saúde do homem. A assistência integral foi citada somente por quatro enfermeiros neste estudo.

$\mathrm{O}$ atendimento integral prevê um olhar sobre a saúde do homem conforme o aspecto biopsicossocial, que está em consonância com o atual conceito de saúde da Organização Mundial da Saúde (Brasil, 2008). Assim, durante a análise, identificou-se que nenhum enfermeiro expôs de maneira completa o objetivo da política.

Um aspecto que chama a atenção, segundo uma entrevistada, está relacionado à ideia de que o MS deveria 'cobrar' ações dos municípios para que efetivamente a PNAISH tivesse êxito. Faz-se necessário refletir sobre o papel dos profissionais de saúde em relação à saúde do homem, pois a expectativa dessa entrevistada é que existisse um sistema de informação que monitorasse a política e as ações desenvolvidas nos municípios. 


\section{Papel do enfermeiro diante da PNAISH}

Os enfermeiros demonstraram que reconhecem o seu papel diante da PNAISH. Onze dos entrevistados relataram ter conhecimento razoável em relação à responsabilidade da enfermagem na implantação dessa política. Sete enfermeiros confirmam ter conhecimento insuficiente, não expondo com clareza o seu papel. Dos entrevistados, nove citam como papel desse profissional a educação e a orientação em saúde, mencionadas na seguinte fala: "O papel é de educação, desmistificação dos preconceitos que eles têm e também ações de prevenção e promoção da saúde" (E2).

Para Santos (2010), a educação em saúde é importante para o cuidado de enfermagem, uma vez que ela pode determinar como os indivíduos e as famílias são capazes de ter comportamentos que conduzam ao autocuidado.

Diante do exposto, faz-se necessário um programa que apresente questões específicas referentes à população masculina. Podem-se citar ações educativas em relação às violências, câncer de próstata, vida sexual e reprodutiva. Também se pode dizer que o MS preocupou-se com essa parcela da população, até então esquecida, implantando a PNAISH.

A mudança do foco programático, saindo do restrito campo da adesão a programas assistenciais de saúde para uma perspectiva mais ampla que privilegie um novo paradigma baseado na atenção integral, valorizou, fundamentalmente, a promoção da saúde e a qualidade de vida, bem como a Educação como importante estratégia para promover mudanças comportamentais indispensáveis à consolidação das ações propostas (Brasil, 2008).

Uma das funções do enfermeiro atuante na atenção básica é capacitar e aperfeiçoar os agentes comunitários de saúde ACSs para trabalhar com a população. Em relação à saúde do homem, os ACSs podem desempenhar ações importantes, que contribuam para a desmistificação dos preconceitos, identificar situações em que há necessidade de busca ativa de pacientes e, de modo geral, fornecer orientações e estimular os homens a procurarem a unidade de saúde.

\section{Fatores que interferem na resistência dos homens em procurar os serviços de saúde}

Ao se analisar a fala dos enfermeiros, percebe-se que as questões culturais representam a principal causa da não adesão dos homens aos serviços de saúde citada por 12 dos enfermeiros entrevistados.

Os resultados desta pesquisa corroboram o estudo de Julião e Weigelt (2011), que afirma que o homem, por uma série de questões culturais e 
educacionais, ainda é visto pela sociedade como uma pessoa invulnerável e forte, livre de qualquer tipo de enfermidade. Isso contribui para que ele não cuide de sua saúde e se exponha mais a riscos que as mulheres.

A busca, pelos homens, de resolução rápida dos seus problemas de saúde faz com que eles procurem alternativas inconsequentes e retarda a procura por serviços de saúde especializados, o que é afirmado na fala a seguir:

Eles vêm quando estão nas últimas, quando já foram para benzedeira, já tomaram chá, já se automedicaram. Daí eles vêm para a unidade. Assim é bem complicado, ele está com um resfriado e está tomando azitromicina, porque foi na farmácia e pegou (E4).

Fagundes e colaboradores (2007) ressaltam que os medicamentos não são mercadorias que possam ser oferecidas de modo igual aos outros bens e serviços. Os medicamentos devem ser utilizados com cuidado, pois sua utilização indevida gera consequências para o paciente. Um fator também evidenciado nas entrevistas diz respeito às barreiras institucionais; estas correspondem às dificuldades enfrentadas ao se acessarem os serviços de saúde e representam uma das principais causas de distanciamento entre os profissionais e a população masculina.

Para haver efetividade na implantação da PNAISH, é necessário que os profissionais se engajem e procurem uma aproximação com a população masculina, estabelecendo vínculos e, de maneira geral, facilitando o acesso aos serviços por essa parcela da população.

\section{Perfil epidemiológico da população masculina segundo os enfermeiros}

Percebeu-se que nenhum dos municípios pesquisados coletou dados para construir um perfil epidemiológico da população masculina. Ou seja, os municípios que estão desenvolvendo ações focadas na saúde do homem não realizaram um estudo epidemiológico prévio. Ratificando esse dado, citam-se as falas de alguns dos entrevistados:

Não, na verdade não temos nenhuma série histórica, até porque esse é um assunto novo, agora que está sendo pensado (E10).

Dados, não. Isso tem com a digitadora, mas infelizmente hoje ela não veio (E9).

Não. A gente não tem nenhum programa para identificar esses dados de saúde do homem, tem da criança, mulher adolescente (E11).

Entendemos que as ações em saúde devem ser realizadas com base no perfil epidemiológico da população, em que haja a identificação das especi- 
ficidades e prioridades pontuais e da região, a fim de se suprir as necessidades da população-alvo.

Destaca-se o depoimento de E11, uma vez que ele diz que há programa para mulher, adolescente, criança, e não há nada referente à saúde do homem. A falta de um programa do MS que compile os dados não justifica a ausência do perfil epidemiológico da população masculina, porque toda e qualquer ação em saúde desenvolvida deve ser embasada pelo conhecimento epidemiológico local.

\section{Recursos financeiros direcionados à saúde do homem}

No que se refere ao repasse de recursos financeiros destinados à saúde do homem, os enfermeiros, em sua maioria (dez), referem não ter conhecimento a respeito, e os demais (sete) responderam que acreditam que não há repasse para o município.

A portaria n. 1.008/2010, que determinou a fração de recursos financeiros para os municípios que implantaram a PNAISH, afirma que disponibilizou R $\$ 75$ mil para cada município escolhido pela Comissão Intergestores Bipartite para serem os pilotos da implantação da política (Brasil, 2010).

Os municípios que assinaram o Pacto pela Saúde tinham ciência de que uma das prioridades era a atenção à saúde do homem. Segundo o MS, por meio do Pacto pela Saúde a responsabilidade de prover recursos para a atenção básica é das três esferas do governo, e eles são repassados do Fundo Nacional de Saúde para o Fundo Municipal de Saúde. Esses recursos financeiros são gastos de acordo com as necessidades dos municípios, conforme o seu planejamento. Assim, no Plano Municipal de Saúde e na Programação Pactuada Integrada podem ser reservados recursos dos recebidos por meio dos pisos da atenção básica, para atender à saúde do homem (Brasil, 2006b).

\section{Ações desenvolvidas nos municípios referentes à saúde do homem}

As ações desenvolvidas nesses municípios são direcionadas à faixa etária a partir dos 40 anos e aos grupos de educação em saúde, como hipertensos e diabéticos, que fazem parte da população com idade mais avançada.

$\mathrm{Na}$ análise das entrevistas, quando mencionadas as consultas médicas e as ações nos grupos de hipertensos e diabéticos, identifica-se que a assistência à saúde está focada no modelo biomédico, o que se contrapõe ao modelo atual de saúde de forma holística/integral proposta pela ESF.

Trindade e colaboradores (2011), em artigo publicado sobre as ações da ESF em um município do interior de Santa Catarina, abordam a sua organização e o modelo de saúde preconizado atualmente na atenção básica: 
O PSF, atualmente chamado de Estratégia Saúde da Família (ESF), tem como intuito fomentar mudanças na política de saúde vigente, por meio da implantação de equipes multidisciplinares que atuam no âmbito da promoção da saúde, prevenção, recuperação, reabilitação de doenças e agravos mais frequentes. Essa estratégia emerge no sentido de organizar a expansão e a qualificação da atenção básica, na busca de superar o antigo modelo assistencial centrado na doença, tendo como princípios fundamentais a integralidade, qualidade da assistência, equidade e participação social (Trindade et al., 2011, p. 163).

O presente estudo corrobora os resultados obtidos por Trindade e colaboradores (2011) no que se refere ao trabalho das equipes da ESF: a atenção básica continua voltada para o modelo biomédico - apesar de existirem alguns trabalhos relacionados de forma multidisciplinar -, a realização da consulta de enfermagem, as atividades de educação em saúde e o trabalho dos ACSs.

Os próprios enfermeiros reconhecem que as ações que eles praticam não são muito efetivas nem específicas para a saúde do homem.

\section{Considerações finais}

Ficou evidenciado neste estudo que há insuficiência de dados e conhecimento sobre a PNAISH por parte dos enfermeiros. Percebe-se a preocupação deles no que diz respeito ao câncer de próstata, pois este é uma das principais ações preventivas desenvolvidas em alguns municípios pertencentes à Amerios. No entanto, não é a principal ação preconizada pelo MS, pois é direcionada para a população acima dos 40 anos de idade, e a PNAISH evidencia que deve ser trabalhada a saúde do homem de maneira integral e que atenda às necessidades da população masculina na faixa etária dos 20 aos 59 anos.

Outro aspecto que deve ser mencionado diz respeito às ações desenvolvidas pelos municípios e que se direcionam ao modelo biomédico em saúde, em que são realizadas atividades em populações que geralmente já têm um agravo estabelecido, ou se atua prevenindo e tratando algumas doenças especificamente, como câncer de próstata, alcoolismo e tabagismo.

Há que se pensar em saúde do homem como no modelo integral, utilizando-se recursos de trabalho multiprofissional e interdisciplinar para romper as barreiras socioculturais e institucionais da não adesão do homem ao serviço de saúde. Entretanto, para Carrara, Russo e Farol (2009, p. 672), essa questão "parece bastante mais complexa do que imaginam os defensores da nova política, pois essa situação paradoxal dos homens em relação a sua própria saúde e ao seu corpo - sua cultura somática - deriva em larga medida da posição que (ainda) ocupam na hierarquia de gênero". Para estes 
autores, a forma como a cultura influencia as relações de poder entre os gêneros favorece a visão paternalista incorporada na política.

Para os homens, articularem reivindicações a partir de uma posição genérica e tornarem-se visíveis como 'homens' significa colocar-se no mesmo plano que as mulheres. Perderiam, assim, a posição de representantes universais da espécie e se arriscariam a perder também suas prerrogativas na hierarquia de gênero.

Os enfermeiros têm a concepção de que a questão cultural é a principal barreira da não adesão dos homens ao serviço de saúde, mas há que se refletir também sobre as questões do acesso ao serviço, que podem constituir uma barreira da não procura por ele, bem como sobre o fato de que os profissionais devem estar aptos a trabalhar com a saúde do homem tendo como base a PNAISH e o perfil de morbimortalidade dessa população.

Em relação a recursos financeiros específicos para essa política, os enfermeiros, em sua maioria, não souberam discorrer sobre a existência deles - o que demonstra desconhecimento sobre a gestão financeira de que eles dispõem para trabalhar na área da saúde.

Os dados demonstraram uma preocupação em relação ao pouco conhecimento dos enfermeiros sobre a PNAISH e ao financiamento do Sistema Único de Saúde. Pode-se inferir a necessidade de um programa de educação continuada oriundo da Secretaria Estadual de Saúde e ou do MS para que os sujeitos desta pesquisa atuem efetivamente na atenção à saúde do homem.

A ESF preconiza que seja trabalhada a saúde da população conforme a realidade local. Os enfermeiros mencionam que há pouca adesão dos homens ao serviço de saúde, que eles não se cuidam, morrem mais que as mulheres.

A atenção básica deve ser eficaz e resolver os problemas de saúde da população, por meio da ESF, e atuar na resolução dos principais agravos a que essa população está exposta, não só no tratamento de doenças como também na promoção da saúde e na prevenção. 
Resumen El estudio buscó conocer la implantación de la Política Nacional de Atención Integral a la Salud del Hombre desde la óptica de los enfermeros. Se trata de un estudio cualitativo, exploratorio y descriptivo, en el que se entrevistaron a 17 enfermeros de los municipios de la Asociación de los Municipios de Entre Ríos, en Santa Catarina (Brasil). Después de analizar los datos, se consideró que hay fragilidades en el conocimiento de la Política Nacional de Atención Integral a la Salud del Hombre, así como en las acciones realizadas en los municipios. La salud del hombre se centra en el modelo biomédico de atención a la salud. Se concluye que hay necesidad de capacitación de los profesionales para el desarrollo de la política de acuerdo con sus premisas.

Palabras clave salud del hombre; políticas públicas; atención básica.

\section{Notas}

1 Graduada em Enfermagem pela Universidade do Estado de Santa Catarina (Udesc), Chapecó, Santa Catarina, Brasil. <daianecristinateixeira@hotmail.com> Correspondência: Rua Benjamin Constant, 84E, CEP 89802-200, Centro, Chapecó, Santa Catarina, Brasil.

2 Especialista em Obstetrícia e Ginecologia pelo Centro Sul-Brasileiro de Pesquisa, Extensão e Pós-Graduação, Joinville, Santa Catarina, Brasil.

$<$ daianekutszepa@hotmail.com>

3 Udesc, Chapecó, Santa Catarina, Brasil. Mestre em Saúde Pública pela Universidade do Oeste de Santa Catarina. <katiadamy@hotmail.com>

4 Udesc, Chapecó, Santa Catarina, Brasil. Mestre em Enfermagem pela Universidade Federal de Santa Catarina. <ivete.krauzer@udesc.br>

5 Resultado parcial do trabalho de conclusão de curso, A política nacional de Atenção Integral à Saúde do homem sob a ótica dos enfermeiros e gestores do SUS, defendido no Curso de Enfermagem da Udesc em 2011. 


\section{Referências}

BRASIL. Ministério da Saúde. Secretaria de Atenção a Saúde. Departamento de Ações Programáticas Estratégicas. Política nacional de atenção integral à saúde do homem. Brasília: MS, 2008. Disponível em: <www. saude.gov./consultapublica $>$. Acesso em: 20 jan. 2011.

BRASIL. Ministério da Saúde. Pacto pela saúde, em defesa da vida e da gestão. Brasília: MS, 2006a. (Série pactos pela saúde). Disponível em: <www.saude.caop.mp.pr.gov.br/arquivos/ File/volumel.pdf $>$. Acesso em: 15 set. 2011.

BRASIL. Ministério da Saúde. Secretária de Atenção Básica. Departamento de Atenção Básica. Política Nacional de Atenção Básica. 4. ed. Brasília: MS, 2006b. Disponível em: <www.saúde.gov.br>. Acesso em: 23 set. 2011.

BRASIL. Ministério da Saúde. Portaria n. 1.008, de 4 de maio de 2010. Expansão da Política Nacional de Atenção Integral à Saúde do Homem por meio de repasse de incentivo financeiro. Ministério da Saúde. Brasília, 2010. Disponível em: <http://bvsms.saude. gov.br/bvs/saudelegis/gm/2010/prt1008_04_ 05_2010.html> Acesso em: 25 ago. 2011.

CARRARA, Sérgio; RUSSO, Jane A; FAROL, Livi. A Política de atenção à saúde do homem: os paradoxos da medicalização do corpo masculino. Physis: Revista de Saúde Coletiva, v. 19, n. 3, p. 659-678, 2009. Disponível em: <www. scielo.br/pdf/physis/v19n3/a06v19n.pdf $>$. Acesso em: 8 jul. 2013.

CONSELHO FEDERAL DE ENFERMAGEM (Cofen). Código de Ética dos profissionais de enfermagem. Rio de Janeiro, 2007. Disponível em: <http://site.portalcofen.gov.br/node/ 4158>. Acesso em: 2 ago. 2011.

CONSELHO NACIONAL DE SECRETÁRIOS DE SAÚDE (Conass). Política Nacional de Atenção Integral a Saúde do Homem. Brasília, 2010. Disponível em: <www.conass. org.br/arquivos/file/nt_072009.pdf>. Acesso em: 20 ago. 2011.
FAGUNDES, Maria J. D. et al. Análise bioética da propaganda e publicidade de medicamentos. Ciência \& Saúde Coletiva. Rio de Janeiro, v. 12, n. 1, p. 221-29, 2007. Disponível em: <http://dx.doi.org/10.1590/ S1413$81232007000100025>$. Acesso em: 26 set. 2011.

JULIÃO, Graziela G.; WEIGELT, Dias L. Atenção à saúde do homem em unidades de Estratégia de Saúde da Família. Revista de Enfermagem da UFSM, Santa Maria, v. 1, n. 2, p. 144-52, 2011. Disponível em: <http:// cascavel.ufsm.br/revistas/ojs-2.2.2/index. php/reufsm/article/viewFile/2400/1743>. Acesso em: 24 set. 2011.

LYRA, Jorge et al. Juventude, mobilização social e saúde: interlocuções com políticas públicas. In: LYRA, Jorge. Homem, jovem, negro e pobre: um novo sujeito para as políticas públicas. Recife: Instituto Papai/MAB/ Canto Jovem, 2010. 136 p. Disponível em: $<$ www.unfpa.org.br/Arquivos/ena.pdf $>$. Acesso em: 4 jul. 2013.

MEDRADO, Benedito; LYRA, Jorge. Por uma matriz feminista de gênero para os estudos sobre homens e masculinidades. Revista Estudos Feministas, v. 16, p. 20-35, 2008. Disponível em: <www.scielo.br/pdf/ref/v16n3/ 05.pdf $>$. Acesso em: 5 jul. 2013.

PINAFO, Elisangela et al. Relações entre concepções e práticas de educação em saúde na visão de uma equipe de saúde da família. Trabalho, Educação Saúde, Rio de Janeiro, v. 9, n. 2, p. 201-221, 2011. Disponível em: $<$ www.revista.epsjv.fiocruz.br/index.php? Area $=$ NumeroAnterior $\&$ Num $=43>$. Acesso em: 12 abr. 2012.

PIROLO, Sueli. M. et al. O desafio de operacionalizar as ações de Atenção Integral à Saúde do Homem na Estratégia Saúde da Família. Faculdade de Medicina de Marília, Secretária Municipal de Saúde de Marília. Marília, 2009. Disponível em: <www.famema.br/pos/petsaude/pet7saudedohomem.pdf $>$. Acesso em: 13 set. 2011. 
REICHERT, Carla D.; ANDRIOLI, Denise C. O conhecimento dos homens sobre o câncer de próstata: uma pesquisa no centro integrado de saúde da família no município de Chapecó, SC. 2010. 78f. Monografia (Bacharel em Enfermagem) - Universidade do Estado de Santa Catarina, Palmitos, 2010. Disponível em: <www.pergamum.udesc.br/dados-bu/ 000000/00000000000F/00000F5B.pdf $>$. Acesso em: 20 set. 2011.

SANTOS, Florinda G. Educação em saúde: o papel do enfermeiro como educador. Franca, 2010. Disponível em: <www.webartigos. com/articles/44521/1/Educacao-em-SaudeO-Papel-do-Enfermeiro-Educador/paginal. html>. Acesso em: 19 set. 2011.
TRINDADE, Letícia L. et al. Reflexões acerca do perfil de atendimento na Estratégia Saúde da Família. Cogitare Enfermagem, Curitiba, v. 16, n.1, p. 162-66, 2011. Disponível em: $<$ http://ojs.c3sl.ufpr.br/ojs2/index.php/ cogitare/article/view/21128/13954>. Acesso em: 29 set. 2011.

Recebido em 25/05/2012

Aprovado em 07/06/2013 